\title{
Treatment of ovarian endometrial cysts in the context of recurrence and fertility
}

\author{
*Izabela Nowak-Psiorz ${ }^{A-D, F}$, ${ }^{*}$ Sylwester M. Ciećwież ${ }^{A-D, F}$, Agnieszka Brodowska ${ }^{A, B, D, F}$, Andrzej Starczewski ${ }^{A, B, D, F}$ \\ Department of Gynecology, Endocrinology and Gynecologic Oncology, Pomeranian Medical University, Szczecin, Poland \\ A - research concept and design; B - collection and/or assembly of data; C - data analysis and interpretation; \\ $D$ - writing the article; $E$ - critical revision of the article; $F$ - final approval of the article
}

\author{
Address for correspondence \\ Sylwester Ciećwież \\ E-mail: s.ciecwiez@scipro.pl \\ Funding sources \\ None declared \\ Conflict of interest \\ None declared \\ * Izabela Nowak-Psiorz and Sylwester Ciećwież \\ contributed equally to this work
}

Received on April 4, 2017

Reviewed on May 6, 2017

Accepted on May 5, 2018

Published online on January 15, 2019

Cite as

Nowak-Psiorz I, CiećwieżS, Brodowska A, Starczewski A.

Treatment of ovarian endometrial cysts in the context of recurrence and fertility. Adv Clin Exp Med. 2019;28(3):407-413. doi:10.17219/acem/90767

DOI

10.17219/acem/90767

\section{Copyright}

Copyright by Author(s)

This is an article distributed under the terms of the

Creative Commons Attribution Non-Commercial License

(http://creativecommons.org/licenses/by-nc-nd/4.0/)

\begin{abstract}
An approach to ovarian endometrial cysts has changed considerably during recent years, especially in regard to treatment of recurrent endometriosis, fertility sparing and infertility management. Surgical treatment is the primary therapeutic option. The most efficient types of treatment are radical procedures involving adhesiolysis, removal of the cyst along with its capsule and any remaining endometriotic foci. However, small asymptomatic cysts should not be treated surgically, especially in patients older than 35 years. Surgical treatment can be considered in infertile women and those who failed to get pregnant despite 1-1.5 years of trials, as well as in cases in which in vitro fertilization is not an option. Also large cysts, with more than $4 \mathrm{~cm}$ in diameter, should be treated surgically due to the risk of their rupture or torsion. The most efficient preventive measure for recurrent ovarian endometriosis is unilateral oophorectomy with sparing the contralateral ovary. Such a procedure should be considered in women who are no longer interested in childbearing or present with another endometriotic cyst in the same ovary. The role of pharmacotherapy is fairly limited; it should be considered in patients in whom diffuse endometriosis is associated with pain. Therapeutic agents from the following groups can be used: estrogen-progestin preparation, gestagens, including progesteronereleasing intrauterine systems and gonadotropin-releasing hormone agonists. Women with infertility should get pregnant as soon as possible, and in patients who failed to get pregnant and/or are older than 35 years, in vitro fertilization should be the treatment of choice.
\end{abstract}

Key words: endometriosis, pharmacotherapy, surgical treatment, endometrioma 


\section{Introduction}

Endometrial ovarian cysts are one of the more common gynecological disorders found in women of reproductive age. They are frequently the cause of surgical interventions, undertaken not only by gynecologists but also by pelvic surgeons. Therefore, at least in this context, endometrial ovarian cysts should be considered an interdisciplinary problem.

Endometriosis is a chronic benign estrogen-dependent disease. It is observed primarily in patients of reproductive age, and its prevalence in this population is estimated at $5-10 \%$. Endometriosis is defined as the presence of active endometrial tissue outside the uterine cavity, usually on the peritoneum of the minor pelvis, in the myometrium, ovaries and fallopian tubes, as well as extraperitoneally. Endometriotic lesions can be also found in the intestines, urinary bladder, lungs, and even in the brain. Based on the localization of the lesions, the disease is classified as peritoneal, ovarian or deep infiltrating endometriosis. ${ }^{1}$

Etiopathogenesis of endometriosis is still not fully understood. There are several theories on the etiology of this condition. The most widely accepted one is Sampson's theory according to which formation of ectopic endometrial tissue is a consequence of retrograde menstruation. During this process, some of the endometrial debris leaves the uterus with small volumes of menstrual blood, reaches abdominal cavity via the fallopian tubes and is implanted into peritoneum, usually within the pelvis. ${ }^{2}$ Furthermore, immune and genetic factors are postulated to play a crucial role in the etiopathogenesis of endometriosis. ${ }^{3}$

The most common manifestations of endometriosis include painful menstrual periods with heavy menstrual bleeding, pelvic pain, dyspareunia, infertility, and sometimes pain during voiding or defecation. Ovarian endometriosis is the most common form of this condition. Ovarian endometrial cysts (endometriomas) are found in $20-55 \%$ of women with endometriosis. ${ }^{4}$

An ovarian mass can be qualified as an endometrial cyst based on its ultrasonographic presentation, using the criteria that have been published by the International Ovarian Tumor Analysis (IOTA) collaboration in 2013. ${ }^{5}$ These criteria include size, shape, echogenicity of the lesion, structure of its capsule, presence of any projections to the cyst's lumen, vasculature, and relationship with surrounding anatomical structures. Currently, differential diagnosis of endometrial cysts and ovarian malignancies can be conducted promptly with the aid of an online application available on the IOTA website. ${ }^{6}$ Another parameter that can be used in the differential diagnosis is the concentration of CA-125, which is usually slightly elevated in patients with this condition. ${ }^{7}$

The approach to ovarian endometrial cysts has changed considerably during recent years, especially regarding the treatment of recurrent endometriosis, fertility sparing and infertility management. Current recommendations of international scientific bodies are based on the results of published meta-analyses and randomized trials.

\section{Watchful waiting}

There is a general consensus that small ovarian endometrial cysts, up to $3 \mathrm{~cm}$ in diameter, should be left untreated. ${ }^{1,8}$ However, 2 questions arise: what is the maximal duration of the expectant management and should it be considered in all patients with endometriosis? With no doubt, the expectant management is excluded in women with pelvic pain; in such cases, surgery is the treatment of choice. ${ }^{9}$ However, is it the same with asymptomatic cases? It was shown that the formation of ovarian endometrial cyst results in a decrease in ovarian reserve, which progresses further if the lesion persists longer or enlarges. This may impair fertility, i.e., due to significantly lower ovulation rate and premature menopause. ${ }^{10}$ Furthermore, the presence of endometrial cysts results in the formation of, frequently massive, solid adhesions between the ovary, fallopian tube and broad ligament of the uterus, which also decrease the likelihood of pregnancy. All this data supports surgical treatment, especially in young women with infertility. ${ }^{11}$ However, surgical treatment is also known to decrease the ovarian reserve, thus promoting reproductive aging and accelerating the onset of menopause, ${ }^{12}$ especially in the case of repeated surgeries. Moreover, one should consider the risk of postoperative adnexal adhesions that may considerably reduce the mobility of the fallopian tubes or result in their complete obliteration.

Treatment of infertile women above 35 years of age should be discussed separately. Surgical management of such patients will result in a decrease in their already low ovarian reserve. In such cases, especially in women with advanced endometriosis, with mechanical or male factor infertility, in vitro fertilization is an efficient option. According to Tsoumpou et al., ${ }^{13}$ surgical treatment of patients prior to implementation of assisted reproductive technologies (ART) does not improve their outcomes. The only exception pertains to large endometrial cysts that hinder access to ovarian follicles. ${ }^{13}$

To summarize, one should be cautious when considering surgical treatment of small ovarian endometrial cysts. Small asymptomatic cysts should not be treated surgically, especially in patients older than 35 years. Surgical treatment should be considered in infertile women and patients who failed to get pregnant despite $1-1.5$ years of trials, as well as in cases in which in vitro fertilization is not an option. ${ }^{8}$ Also large cysts, with more than $4 \mathrm{~cm}$ in diameter, should be treated surgically due to the risk of their rupture or torsion.

\section{Surgical treatment}

Surgical treatment is the principal method of endometrial cyst management. The least efficient technique, nowadays considered mostly obsolete, involves puncturing the cyst under ultrasonographic guidance or during 
laparoscopy, aspiration of its contents, irrigation and, whenever necessary, administration of a sealant. However, this procedure frequently led to complications, such as the formation of abscesses and peritoneal adhesions. Moreover, up to $80-90 \%$ of patients present with recurrence already 6 months after the procedure. ${ }^{14}$ Another surgical procedure, still used in many centers, is a cystectomy with irrigation of the lumen of the cyst and coagulation of its capsule. ${ }^{15}$ Bipolar coagulation, optimally argon plasma coagulation, or laser coagulation should be used to spare normal ovarian tissue. ${ }^{16}$ Unfortunately, this technique also does not guarantee the complete destruction of the capsule of the cyst. Saleh and Tulandi ${ }^{14}$ showed that more than a half of patients presented with recurrent cysts as early as 2 years after the procedure, and 42 months post-surgery the recurrence rate increased to $60 \%$.

Currently, laparoscopy is considered the "gold standard" in the management of ovarian endometrial cysts. The laparoscopic procedure includes freeing the ovary from adhesions, cystectomy, irrigation of the cyst, and complete excision of its wall with least possible injury to normal ovarian tissue. Bleeding vessels should be coagulated with a bipolar electrode, optimally using argon plasma coagulation. Also, all other endometriotic foci present in the pelvis should be coagulated carefully whenever feasible. ${ }^{17,18}$ The abovementioned technique is associated with the highest pregnancy rates in patients with infertility ${ }^{18,19}$ Moreover, the removed ovarian cyst can be subjected to a histopathologic examination, which is of vital importance, considering that ca. $0.8-0.9 \%$ of endometriomas turn out to be malignant. In one study, up to $13 \%$ of endometriomas were eventually identified as borderline ovarian tumors. ${ }^{20}$ The ovarian-sparing treatment with complete removal of the capsule of the cyst results in a considerable decrease in the endometrioma recurrence rate. However, between $10 \%$ and $40 \%$ of the patients may present with recurrent cysts, and the recurrence rate was shown to increase with the time elapsed since surgery. ${ }^{21,22}$

The severity of endometriosis turned out to be a key determinant of sustained remission after surgical treatment. The severity of the disease is determined with revised American Society for Reproductive Medicine (rASRM) scoring system, and many previous studies showed that the higher the rASRM score and severity of endometriosis, the greater the risk of its recurrence. ${ }^{21,23,24}$ Liu et al. showed that the rASRM severity grade correlates with the recurrence rate of endometriosis, but its prognostic value is relatively poor. ${ }^{25}$ However, this hypothesis is not supported by all the authors. For example, Koga et al. did not find a significant association between the severity of endometriosis and its recurrence rate. ${ }^{26}$ Yun et al. analyzed the role of various components of the rASRM scoring system as potential risk markers for recurrent endometriosis. ${ }^{27}$ They showed that the risk of recurrence increases considerably in patients with adhesions involving the ovaries and/or fallopian tubes and/or with complete obliteration of the cul-de-sac. ${ }^{27}$ Consequently, the presence of adhesions, especially involving adnexa and culde-sac, seems to be an important predictor of recurrence. This hypothesis was also confirmed by other authors. ${ }^{24,25,27}$

Bilateral endometrial cysts are generally considered to be a prognostic factor of recurrence, although not all previous studies confirmed this relationship. ${ }^{26}$ An association between the localization of the cyst and the risk of recurrence is unclear. However, the results of some studies suggest that endometriomas located in the left ovary are more likely to recur. ${ }^{28}$ Also, the available data on the influence of the diameter of the cyst on the recurrence rate is inconclusive. Nevertheless, a larger diameter of the cyst is generally considered an unfavorable prognostic factor. ${ }^{21,26,29}$ However, a minimum diameter of the lesion associated with unfavorable prognosis has not been established thus far. Moreover, Ghezzi et al. showed that the diameter of the cyst has no influence on the recurrence rate. ${ }^{28}$ Similar findings were also reported by Porpora et al. ${ }^{24}$

Similarly, no consensus was reached regarding the influence of the patient's age on the risk of recurrence. According to many researchers, young age has an unfavorable effect on the duration of remission after surgical treatment. This is probably related to the fact that younger women present with more aggressive forms of endometriosis and higher postoperative blood concentrations of estrogens. ${ }^{21,25} \mathrm{How}-$ ever, Koga et al. ${ }^{26}$ did not find an association between the patient's age and the duration of remission, and according to Parazzini et al., ${ }^{30}$ the risk of recurrence increases with age.

Pregnancy was shown to exert a beneficial effect and to reduce the risk of recurrent endometriosis, and as such is even considered a protective factor. Elevated concentrations of progesterone in pregnancy may inhibit the growth of endometriotic foci and attenuate related inflammation. ${ }^{23,31}$ A relatively less often considered prognostic factor of recurrent endometriosis is the preoperative level of CA-125. A high preoperative level of CA-125 was postulated to predispose to recurrence. Due to its low specificity, this antigen is not useful as a diagnostic marker of the disease, but still may play an important role in the monitoring of its treatment. ${ }^{7}$ In turn, no associations were found between the presence of uterine leiomyoma or adenomyosis and recurrence rate. $^{26}$

Nowadays, molecular background of endometriosis is a subject of ongoing research. Considering the estrogen-dependence of the disease, estrogen receptor (ER), present in 2 forms, ER- $\alpha$ and ER- $\beta$, was an obvious target of research. Some studies showed an association between ER- $\alpha$ polymorphism and the risk of recurrence. ${ }^{32}$ Another risk marker for recurrent endometriosis may be cyclooxygense-2. Increased activity of this enzyme, catalyzing synthesis of prostaglandins, was observed in endometriotic lesions in women who later presented with recurrence. ${ }^{33}$

The most efficient preventive measure for recurrent ovarian endometriosis is unilateral oophorectomy with sparing the contralateral ovary. Such a procedure should 
be considered in women who are no longer interested in childbearing or present with another endometriotic cyst in the same ovary. Importantly, the procedure should be extended to adhesiolysis and the removal of all remaining endometriotic foci. Namnoum et al. ${ }^{34}$ demonstrated that residual adhesions and endometriotic foci are associated with an 8-fold increase in reoperation risk. Hysterectomy with the removal of adnexa can be an option in perimenopausal women with endometrial cysts, diffuse peritoneal endometriosis or deep infiltrating endometriosis, especially with concomitant pain. ${ }^{35}$

\section{Pharmacotherapy}

Currently, pharmacotherapy is considered to play a secondary role during the postoperative period, and as such is implemented only in selected cases. Furthermore, preoperative pharmacotherapy was shown to exert an unfavorable effect on ovarian endometrial cysts. Koga et al. ${ }^{26}$ demonstrated that pharmacotherapy constitutes a risk factor for postoperative recurrence, since causing atrophy and fibrosis may hinder intraoperative identification of all endometriotic foci. In such cases, resection is more likely to be incomplete and is more technically demanding. ${ }^{26}$ Similar conclusions were also reported by Muzii et al. ${ }^{36}$ Moreover, preoperative pharmacotherapy was shown to be associated with an increase in the proportion of dyskariotic cells and to slow down development of eukaryotic cells within the cyst's wall. ${ }^{37}$

Postoperative pharmacotherapy is recommended after incomplete resection of endometriotic foci or in patients with concomitant pain. Endometriosis is an estrogen-dependent condition and, therefore, the principal aim of pharmacotherapy is to suppress ovarian function and to promote the atrophy of endometriotic lesions. Therapeutic agents from the following groups can be used: estrogenprogestin preparation, gestagens, including progesteronereleasing intrauterine systems and gonadotropin-releasing hormone $(\mathrm{GnRH})$ agonists. Due to its multiple side effects, danazol is no longer recommended in pharmacotherapy of endometriosis. ${ }^{38}$ Frequently, the agents mentioned above are simultaneously used as contraceptives, and, therefore, specific agents should be selected on an individual basis with their potential side effects taken into consideration.

Two-component oral contraceptives play an important role in the prevention of recurrent endometriotic lesions and the attenuation of pain. Beneficial effects of these preparations are associated with the inhibition of ovulation. Less intensive menstrual bleeding and lack of retrograde menstruation prevent endometrial debris spreading outside the uterus. Furthermore, estroprogestin contraceptives suppress the proliferation of endometriotic tissue. Their superiority to other therapeutic options is associated with their small number of side effects, good tolerance and reasonable price.
Due to the frequent use of oral contraceptives, their efficacy was a subject of many previous studies. During a 24-month postoperative follow-up, Seracchioli et al. ${ }^{39}$ found recurrent endometriomas in $29 \%$ of women who did not use any contraceptives, as well as in $14.7 \%$ and $8.2 \%$ of patients who used cyclic and continuous contraceptives, respectively. The same group showed that the diameter of recurrent endometrial cysts in women who used hormonal preparations was markedly smaller than in patients who did not receive this form of therapy. Also, the growth rate of recurrent lesions in the former group was reduced. The most beneficial effects were observed in patients taking continuous contraceptives. ${ }^{40,41}$ Zorbas et al. ${ }^{42}$ compared the effects of continuous and cyclic contraceptives. The risk of recurrence turned out to be markedly higher in patients using cyclic contraceptives (16.6\%) than in those undergoing continuous therapy, probably due to the fact that the latter resulted in constant suppression of inflammatory processes. ${ }^{42}$ Cucinella et al ${ }^{43}$ analyzed the efficacy of oral contraceptives depending on the type of progestin they contained. Contrary to other authors, they showed that the type of progestin (desogestrel, gestodene, dienogest) exerted no effect on the efficacy of oral contraceptives in the prevention of recurrent endometriosis. In contrast, the results of other studies point to more beneficial effects of dienogest, but this may be a consequence of its synergy with endogenous estradiol. ${ }^{44}$ Surprisingly, however, Muzii et al. ${ }^{45}$ showed that postoperative administration of low-dose contraceptives does not exert a significant effect on the recurrence rate of endometriosis, ${ }^{45}$ but may only prolong the time to recurrence. The absence of significant effects of treatment with contraceptives on the recurrence rate of endometriosis was also reported by Koga et al. ${ }^{26}$ However, the latter authors analyzed the effects of shortterm administration of oral contraceptives ( $<12$ months) and it is generally believed that it is the long-term therapy that shows the most evident benefits. Furthermore, the protective effect of oral contraceptives dramatically decreases after their withdrawal. Consequently, continuation of the therapy is recommended until the patient decides to get pregnant. ${ }^{24,31}$

Of all gestagens, dienogest is used is monotherapy most often. It inhibits the growth of endometriotic foci, markedly attenuates pain and rarely produces side effects when administered at $2 \mathrm{mg}$ per day. ${ }^{44,46}$ The results of previous studies suggest that dienogest therapy may also result in a lower recurrence rate of endometriosis. According to Yanase et al., ${ }^{47}$ a 6-month cyclic therapy with this gestagen after previous surgical treatment of endometriosis protects against the recurrence of the disease, and this protective effect may last for approx. 4 years. Similar findings were also reported by Ouchi et al. ${ }^{22}$

Also levonorgestrel-releasing intrauterine systems found an application in the adjuvant treatment of endometriosis. Their principal advantages include the possibility to achieve and maintain high local concentrations of levonorgestrel 
without concomitant suppression of the ovary. The system turned out to be particularly effective in the control of pain, but its role in the prevention of recurrent endometriosis is put into question. The only documented beneficial effect was a longer time to recurrence. ${ }^{48}$ Another study compared the efficacy of a levonorgestrel-releasing intrauterine system and dienogest-containing oral contraceptives. While the latter turned out to be markedly more effective in attenuating pain, they were only slightly more effective in preventing recurrence. ${ }^{49}$

GnRH analogues are another group of therapeutics that have been tested for their potential application in the prevention of recurrent endometriosis. Their beneficial effects were observed if they had been administered for at least 6 months post-surgery. However, a 3-month therapy exerted a markedly less pronounced effect on the recurrence rates. Aside from reducing the recurrence rate, $\mathrm{GnRH}$ analogues were also shown to prolong the time to recurrence. However, the potential side effects of the therapy need to be emphasized, as it was shown that even a 6-month therapy with $\mathrm{GnRH}$ analogues may promote bone demineralization. ${ }^{50}$

Aromatase inhibitors are a novel group of therapeutics. Alborzi et al. ${ }^{51}$ compared the efficacy of aromatase inhibitors and GnRH analogues in the prevention of recurrent endometriosis after laparoscopic treatment. Both agents were administered for 2 months, and then the patients were followed up for another 12 months. The recurrence rate in patients treated with aromatase inhibitors was similar as in women who received GnRH analogues. Surprisingly, a similar recurrence rate was also observed in the controls (patients without any pharmacotherapy). However, it is the duration of the therapy which probably has a considerable effect on its outcome.

\section{Management of endometrial cysts in women with infertility}

Management of ovarian endometrial cysts in women with infertility should be discussed separately. As mentioned above, endometrial cysts undoubtedly compromise fertility. ${ }^{52-54}$ On the other hand, surgical treatment is associated with a decrease in ovarian reserve and the induction of peritoneal adhesions. Concentration of anti-Müllerian hormone was demonstrated to decrease markedly immediately after surgery and remain at a lower level for 6-9 months. ${ }^{55,56}$ Surgical treatment results in a permanent, ca. $10 \%$, decrease in antral follicle count. ${ }^{57}$ The influence of surgical treatment on ovarian reserve is particularly evident in the case of cysts larger than $4 \mathrm{~cm}$ in diameter. ${ }^{57,58}$ Consequently, surgical treatment is not recommended in patients with small cysts and in women older than 35 years. Such patients should get pregnant as soon as possible, and whenever needed they can be subjected to the induction of ovulation and intrauterine insemination, ${ }^{8}$ although the effectiveness of the latter procedure in this setting is often put into question. ${ }^{59,60}$ In patients who failed to get pregnant and in women older than 35 years, in vitro fertilization is the treatment of choice. ${ }^{8,59,60}$ Surgical treatment should be limited solely to the large cysts that may hinder ovarian puncture, ${ }^{13}$ and to the lesions being suspicious for malignancy based on their ultrasonographic appearance.

\section{Take-home message}

In this paper, we have reviewed the results of studies conducted at various centers over a 30-year period, to demonstrate the efficacy of the treatment for ovarian endometrial cysts in the context of their recurrence. To the best of our knowledge, the number of similar reviews is sparse, although such publications would with no doubt raise the awareness of the appropriate treatment for this most common type of endometriosis among gynecologists, and would support them in the selection of the most effective therapeutic option. Still, coagulation of the capsule of the endometrial cyst with the state-of-the-art devices, such as lasers or plasma knifes, is preferred at some centers, without consideration of recurrence rates after such procedures. The published data and our own clinical experiences suggest that surgery should constitute the primary treatment for ovarian endometriosis. The most efficient types of surgery are radical procedures involving adhesiolysis, removal of the cyst along with its capsule and any remaining endometriotic foci. The role of pharmacotherapy is fairly limited; it should be considered in patients in whom diffuse endometriosis is associated with pain. Women with infertility should get pregnant as soon as possible, and in patients who failed and/or are older than 35 years, in vitro fertilization should be the treatment of choice.

\section{References}

1. Leyland N, Casper R, Laberge P, Singh SS; SOGC. Endometriosis: Diagnosis and management. J Obstet Gynaecol Can. 2010;32(7 Suppl 2): S1-S32.

2. Jiang QY, Wu RJ. Growth mechanisms of endometriotic cells in implanted places: A review. Gynecol Endocrinol. 2012;28(7):562-567.

3. Yang CW, Chang CY, Lai MT, et al. Genetic variations of MUC17 are associated with endometriosis development and related infertility. BMC Med Genet. 2015;16:015-0209.

4. Hickey M, Ballard K, Farquhar C. Endometriosis. BMJ. 2014;19.

5. Sayasneh A, Wynants L, Preisler J, et al. Multicentre external validation of IOTA prediction models and RMI by operators with varied training. Br J Cancer. 2013;108(12):2448-2454.

6. Van Calster B, Van Hoorde K, Froyman W, et al. Practical guidance for applying the ADNEX model from the IOTA group to discriminate between different subtypes of adnexal tumors. Facts Views Vis Obgyn. 2015;7(1):32-41.

7. Szubert M, Suzin J, Wierzbowski T, Kowalczyk-Amico K. CA-125 concentration in serum and peritoneal fluid in patients with endometriosis - preliminary results. Arch Med Sci. 2012;8(3):504-508.

8. Dunselman GA, Vermeulen N, Becker C, et al; European Society of Human Reproduction and Embryology. ESHRE guideline: Management of women with endometriosis. Hum Reprod. 2014;29(3): 400-412. 
9. Schliep KC, Mumford SL, Peterson CM, et al. Pain typology and incident endometriosis. Hum Reprod. 2015;30(10):2427-2438.

10. Uncu G, Kasapoglu I, Ozerkan K, Seyhan A, Oral Yilmaztepe A, Ata B. Prospective assessment of the impact of endometriomas and their removal on ovarian reserve and determinants of the rate of decline in ovarian reserve. Hum Reprod. 2013;28(8):2140-2145.

11. Ofer A, Shulman LP, Singh SS. Improving the Treatment and Management of Endometriosis: Improving the treatment and management of endometriosis: An overview of current and novel approaches. Am J Obstet Gynecol. 2015;19:02543-02540.

12. Coccia ME, Rizzello F, Mariani G, Bulletti C, Palagiano A, Scarselli G. Ovarian surgery for bilateral endometriomas influences age at menopause. Hum Reprod. 2011;26(11):3000-3007.

13. Tsoumpou I, Kyrgiou M, Gelbaya TA, Nardo LG. The effect of surgical treatment for endometrioma on in vitro fertilization outcomes: A systematic review and meta-analysis. Fertil Steril. 2009;92(1):75-87.

14. Saleh A, Tulandi T. Reoperation after laparoscopic treatment of ovarian endometriomas by excision and by fenestration. Fertil Steril. 1999;72(2):322-324

15. Golfier F, Sabra M. Surgical management of endometriosis [in French]. J Gynecol Obstet Biol Reprod. 2007;36(2):162-172.

16. Fayez JA, Vogel MF. Comparison of different treatment methods of endometriomas by laparoscopy. Obstet Gynecol. 1991;78(4):660-665.

17. Giudice LC, Kao LC. Endometriosis. Lancet. 2004;364(9447):1789-1799.

18. Hart RJ, Hickey M, Maouris $P$, et al. Excisional surgery versus ablative surgery for ovarian endometriomata. Cochrane Database Syst Rev. 2008;16(2):CD004992.

19. Alborzi S, Momtahan M, Parsanezhad ME, Dehbashi S, Zolghadri J, Alborzi S. A prospective, randomized study comparing laparoscopic ovarian cystectomy versus fenestration and coagulation in patients with endometriomas. Fertil Steril. 2004;82(6):1633-1637.

20. Stern RC, Dash R, Bentley RC, Snyder MJ, Haney AF, Robboy SJ. Malignancy in endometriosis: Frequency and comparison of ovarian and extraovarian types. Int J Gynecol Pathol. 2001;20(2):133-139.

21. Moini A, Arabipoor A, Ashrafinia N. Risk factors for recurrence rate of ovarian endometriomas following a laparoscopic cystectomy. Minerva Med. 2014;105(4):295-301.

22. Ouchi N, Akira S, Mine K, Ichikawa M, Takeshita T. Recurrence of ovarian endometrioma after laparoscopic excision: Risk factors and prevention. J Obstet Gynaecol Res. 2014;40(1):230-236.

23. Li HJ, Leng JH, Lang JH, et al. Correlative factors analysis of recurrence of endometriosis after conservative surgery [in Chinese]. Zhonghua Fu Chan Ke Za Zhi. 2005:40(1):13-16.

24. Porpora MG, Pallante D, Ferro A, Crisafi B, Bellati F, Benedetti Panici P. Pain and ovarian endometrioma recurrence after laparoscopic treatment of endometriosis: A long-term prospective study. Fertil Steril. 2010;93(3):716-721.

25. Liu X, Yuan L, Shen F, Zhu Z, Jiang H, Guo SW. Patterns of and risk factors for recurrence in women with ovarian endometriomas. Obstet Gynecol. 2007;109(6):1411-1420.

26. Koga K, Takemura Y, Osuga Y, et al. Recurrence of ovarian endometrioma after laparoscopic excision. Hum Reprod. 2006;21(8):2171-2174.

27. Yun $\mathrm{BH}$, Jeon $\mathrm{YE}$, Chon SJ, et al. The prognostic value of individual adhesion scores from the revised American Fertility Society Classification System for Recurrent Endometriosis. Yonsei Med J. 2015;56(4):1079-1086.

28. Ghezzi F, Beretta P, Franchi M, Parissis M, Bolis P, Recurrence of ovarian endometriosis and anatomical location of the primary lesion. Fertil Steril. 2001;75(1):136-140.

29. Guzel Al, Topcu HO, Ekilinc S, et al. Recurrence factors in women underwent laparoscopic surgery for endometrioma. Minerva Chir. 2014;69(5):277-282.

30. Parazzini F, Bertulessi C, Pasini A, et al; Gruppo Italiano di Studio Endometriosi.. Determinants of short term recurrence rate of endometriosis. Eur J Obstet Gynecol Reprod Biol. 2005;121(2):216-219.

31. Guo SW. Recurrence of endometriosis and its control. Hum Reprod Update. 2009;15(4):441-461.

32. Luisi S, Galleri L, Marini F, Ambrosini G, Brandi ML, Petraglia F. Estrogen receptor gene polymorphisms are associated with recurrence of endometriosis. Fertil Steril. 2006;85(3):764-766.

33. Yuan L, Shen F, Lu Y, Liu X, Guo SW. Cyclooxygenase-2 overexpres sion in ovarian endometriomas is associated with higher risk of recurrence. Fertil Steril. 2009;91(4 Suppl):1303-1306
34. Namnoum AB, Hickman TN, Goodman SB, Gehlbach DL, Rock JA. Incidence of symptom recurrence after hysterectomy for endometriosis. Fertil Steril. 1995;64(5):898-902.

35. Chalermchockchareonkit A, Tekasakul P, Chaisilwattana P, Sirimai K, Wahab N. Laparoscopic hysterectomy versus abdominal hysterectomy for severe pelvic endometriosis. Int J Gynaecol Obstet. 2012;116(2):109-111.

36. Muzii L, Marana R, Caruana P, Mancuso S. The impact of preoperative gonadotropin-releasing hormone agonist treatment on laparoscopic excision of ovarian endometriotic cysts. Fertil Steril. 1996;65(6):1235-1237.

37. Blumenfeld Z. Hormonal suppressive therapy for endometriosis may not improve patient health. Fertil Steril. 2004;81(3):487-492.

38. Selak V, Farquhar C, Prentice A, Singla A. Danazol for pelvic pain associated with endometriosis. Cochrane Database Syst Rev. 2007:17(4):CD000068..

39. Seracchioli R, Mabrouk M, Frasca C, et al. Long-term cyclic and continuous oral contraceptive therapy and endometrioma recurrence: A randomized controlled trial. Fertil Steril. 2010;93(1):52-56.

40. Crosignani $P$, Olive D, Bergqvist A, Luciano A. Advances in the management of endometriosis: An update for clinicians. Hum Reprod Update. 2006;12(2):179-189.

41. Vercellini P, Somigliana E, Daguati R, Vigano P, Meroni F, Crosignani PG. Postoperative oral contraceptive exposure and risk of endometrioma recurrence. Am J Obstet Gynecol. 2008;198(5):504.e1-5.

42. Zorbas KA, Economopoulos KP, Vlahos NF. Continuous versus cyclic oral contraceptives for the treatment of endometriosis: A systematic review. Arch Gynecol Obstet. 2015;292(1):37-43.

43. Cucinella G, Granese R, Calagna G, et al. Oral contraceptives in the prevention of endometrioma recurrence: Do the different progestins used make a difference? Arch Gynecol Obstet. 2013;288(4):821-827.

44. Schindler AE. Dienogest in long-term treatment of endometriosis. Int J Womens Health. 2011;3:175-184.

45. Muzii L, Marana R, Caruana P, Catalano GF, Margutti F, Panici PB. Postoperative administration of monophasic combined oral contraceptives after laparoscopic treatment of ovarian endometriomas: A prospective, randomized trial. Am J Obstet Gynecol. 2000;183(3):588-592.

46. Koga K, Takamura M, Fujii T, Osuga Y. Prevention of the recurrence of symptom and lesions after conservative surgery for endometriosis. Fertil Steril. 2015;104(4):793-801.

47. Yanase T, Ishida M, Nishijima S, et al. Outcomes of treatment with cyclic administration of dienogest after ovarian endometriotic cystectomy. Gynecol Endocrinol. 2015;31(8):643-646.

48. Cho S, Jung JA, Lee $Y$, et al. Postoperative levonorgestrel-releasing intrauterine system versus oral contraceptives after gonadotropinreleasing hormone agonist treatment for preventing endometrioma recurrence. Acta Obstet Gynecol Scand. 2014;93(1):38-44.

49. Morelli M, Sacchinelli A, Venturella R, Mocciaro R, Zullo F. Postoperative administration of dienogest plus estradiol versus levonorgestrel-releasing intrauterine device for prevention of pain relapse and disease recurrence in endometriosis patients. J Obstet Gynaecol. 2013;39(5):985-990.

50. Jee BC, Lee JY, Suh CS, Kim SH, Choi YM, Moon SY. Impact of GnRH agonist treatment on recurrence of ovarian endometriomas after conservative laparoscopic surgery. Fertil Steril. 2009;91(1):40-45.

51. Alborzi S, Hamedi B, Omidvar A, Dehbashi S, Alborzi S, Alborzi M. A comparison of the effect of short-term aromatase inhibitor (letrozole) and GnRH agonist (triptorelin) versus case control on pregnancy rate and symptom and sign recurrence after laparoscopic treatment of endometriosis. Arch Gynecol Obstet. 2011;284(1):105-110.

52. Georgievska J, Sapunov S, Cekovska S, Vasilevska K. Effect of two laparoscopic techniques for treatment of ovarian endometrioma on ovarian reserve. Med Arch. 2015;69(2):88-90.

53. Kitajima M, Defrere S, Dolmans MM, et al. Endometriomas as a possible cause of reduced ovarian reserve in women with endometriosis. Fertil Steril. 2011;96(3):685-691.

54. Seyhan A, Ata B, Uncu G. The impact of endometriosis and its treatment on ovarian reserve. Semin Reprod Med. 2015;33(6):422-428.

55. Biacchiardi CP, Piane LD, Camanni M, et al. Laparoscopic stripping of endometriomas negatively affects ovarian follicular reserve even if performed by experienced surgeons. Reprod Biomed Online. 2011;23(6):740-746. 
56. Hwu YM, Wu FS, Li SH, Sun FJ, Lin MH, Lee RK. The impact of endometrioma and laparoscopic cystectomy on serum anti-Mullerian hormone levels. Reprod Biol Endocrinol. 2011;9:1477-7827.

57. Urman B, Alper E, Yakin K, et al. Removal of unilateral endometriomas is associated with immediate and sustained reduction in ovarian reserve. Reprod Biomed Online. 2013;27(2):212-216.

58. Tang Y, Chen SL, Chen X, et al. Ovarian damage after laparoscopic endometrioma excision might be related to the size of cyst. Fertil Steril. 2013;100(2):464-469.
59. Kuczyński W, Kurzawa R, Oszukowski P; Polish Gynecological Society and Polish Society for Reproductive Medicine. Polish Gynecological Society and Polish Society for Reproductive Medicine recommendations for the diagnosis and treatment of infertility [in Polish]. Ginekol Pol. 2012;83(2):149-154.

60. National Institute for Health and Clinical Excellence. Fertility problems: Assessment and treatment. Clinical guideline. National Institute for Health and Clinical Excellence. http://niceorguk/guidance/ cg156. Accessed February 5, 2017. 